\title{
Usefulness of Stabilizer for Microanastomoses of Internal Thoracic Vessels during DIEP Flap Breast Reconstruction
}

\author{
Atsumori Hamahata, MD, $\mathrm{PhD}^{1}$ Kazuyuki Kubo, $\mathrm{MD}^{2}$ Hiroshi Matsumoto, MD, PhD \\ Takashi Yamaki, MD, $\mathrm{PhD}^{3}$ Hiroyuki Sakurai, MD, $\mathrm{PhD}^{3}$ \\ ${ }^{1}$ Division of Plastic and Reconstructive Surgery, Saitama Cancer \\ Center, Saitama, Japan \\ ${ }^{2}$ Division of Breast Surgery, Saitama Cancer Center, Saitama, Japan \\ ${ }^{3}$ Department of Plastic and Reconstructive Surgery, Tokyo Women's \\ Medical University, Tokyo, Japan \\ Address for correspondence Atsumori Hamahata, MD, PhD, Division \\ of Plastic and Reconstructive Surgery, Saitama Cancer Center, 780 \\ Komuro Inamachi Kitaadachigunn Saitama, Saitama, Japan \\ (e-mail: a.hamahata@cancer-c.pref.saitama.jp).
}

J Reconstr Microsurg Open 2016;1:26-28.

\begin{abstract}
Background Internal mammary arteries and veins are some of the most useful recipient vessels for autologous breast reconstruction. However, the internal mammary vessels are relatively small, which requires a high magnification power during microanastomoses, and respiratory fluctuations often disturb surgeons' focus on the vessels and affect performance of precise microanastomoses. Therefore, we developed a stabilizer for use during deep inferior epigastric artery perforator (DIEP) flap breast reconstructions.

Methods From April 2013 to March 2015, 23 patients underwent DIEP flap breast reconstructions. In 15 of the patients (22 venous anastomoses, 15 arterial anastomoses), the microanastomoses were performed without the stabilizer. In eight of the patients (10 venous anastomoses, 8 arterial anastomoses), the microanastomoses were performed with the stabilizer. Times for each microanastomosis procedure (from the first suture to the last suture) were measured using retrospective intraoperative video in the two groups.

Results All flaps survived without any microanastomosis problems. The average arterial microanastomosis times were 13 minutes 56 seconds (SD, 0 minute 53 seconds) in the stabilizer group and 15 minutes 25 seconds (SD, 4 minutes 11 seconds) in the nonstabilizer group $(p<0.05)$. The average venous microanastomosis times

Keywords

- stabilizer

- respiratory fluctuation

- breast reconstruction were 16 minutes 21 seconds (SD, 2 minutes 4 seconds) in the stabilizer group and 22 minutes 16 seconds (SD, 2 minutes 8 seconds) in the nonstabilizer group $(p<0.05)$. Conclusion We developed a stabilizer for microanastomosis of internal mammary vessels during DIEP flap breast reconstruction. The stabilizer significantly decreased the time of microanastomosis and reduced microsurgeons' stress.
\end{abstract}

The lower abdominal region is the most useful donor site for breast reconstruction. After Holmström and Robbins described the free transverse rectus abdominis myocutaneous flap, Koshima and Soeda transformed the flap into the deep inferior epigastric artery perforator (DIEP) flap. ${ }^{1-3}$ The DIEP flap has become popular for breast reconstruction through- out the world. However, free flap transfer requires microanastomoses, and complications remain associated with that procedure. When transferring abdominal tissue to the breast, internal mammary vessels or thoracodorsal vessels are commonly used as the recipient vessels. However, the use of the internal mammary vessels has been reported to be clearly received

November 9, 2015 accepted after revision December 21, 2015 published online April 18, 2016
DOI http://dx.doi.org/ $10.1055 / \mathrm{s}-0036-1571845$ ISSN 2377-0813.
Copyright (c) 2016 by Thieme Medical Publishers, Inc., 333 Seventh Avenue, New York, NY 10001, USA. Tel: +1(212) 584-4662.
License terms

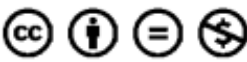


justified and preferred because of its highly pressurized system, consistent location, ease of accessibility, and flap positioning. ${ }^{4}$ However, the vessels are small and require microanastomoses under high magnification power, and respiratory fluctuations often disturb surgeons' focus. Respiratory fluctuation causes continuous focus field shifts and could be one reason for decreased quality of precise microanastomoses. In addition, these surgeons commonly suffer from asthenopia and in extreme cases experience optic vertigo. To reduce respiratory fluctuation during microsurgery, we have developed a stabilizer and tested its usefulness.

\section{Materials and Methods}

From April 2013 to March 2015, 23 patients (average age: 48.7 years; standard deviation $[\mathrm{SD}]=7.2$ years) in Saitama Cancer Center (Saitama, Japan) underwent breast reconstruction using DIEP flap. Internal mammary vessels were used as recipient vessels in all cases. In the first 15 patients, the stabilizer was not used during microanastomosis (15 arterial anastomoses and 22 venous anastomoses). In the final eight patients, the stabilizer was used during microanastomosis ( 8 arterial anastomoses and 10 venous anastomoses). Times for each microanastomosis procedure (from first suture to last suture) were measured using retrospective intraoperative video in the two groups. The diameter of the vessels was also measured. Summary statistics of data are expressed as means (SD). Statistical comparison was performed by unpaired $t$-test.

\section{Microanastomosis with Stabilizer}

The tip (15 mm length) of a $15-\mathrm{mm}$ wide flexible retractor spatula (Mizuho Corporation, Tokyo, Japan) was bent to 90 degrees for insertion into the space where the third costal rib was removed. The retractor spatula was attached to the Octopus universal retractor holder (Yufu Itonaga Co. Ltd., Tokyo, Japan). After elevating the DIEP flap and preparing the internal mammary artery and vein, the tip of retractor spatula, which we call the "stabilizer," was placed under the internal mammary vessel and deep inferior epigastric vessel. The Octopus universal holder could be flexed freely and the tip of retractor spatula could be adjusted to any position ( - Figs. 1 and 2). After the tip of retractor spatula was positioned $1 \mathrm{~cm}$ above the pleura, microanastomoses were performed on the stabilizer using a high-resolution microscope ( - Video 1 ).

\section{Video 1}

The microscopic view without and with the stabilizer. Online content including video sequences viewable at: https://www.thieme-connect.com/products/ ejournals/html/10.1055/s-0036-1571845.

\section{Results}

All flaps survived without any microanastomosis problems. The average arterial microanastomosis times were

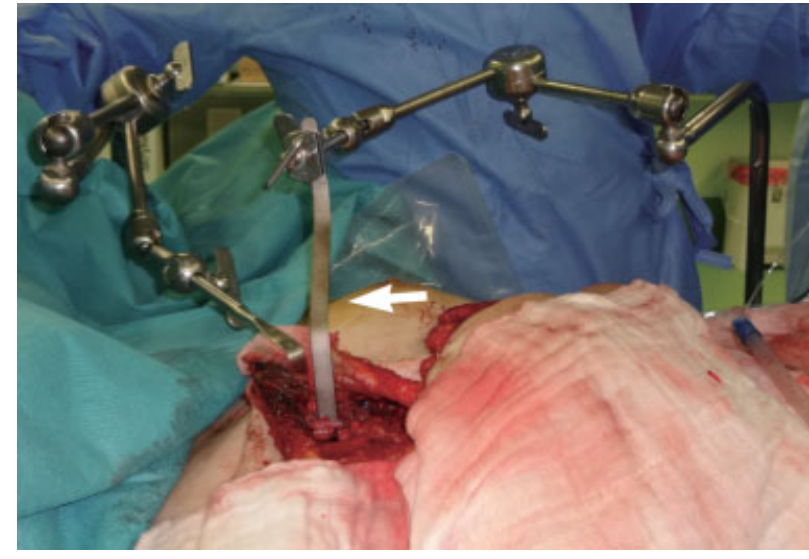

Fig. 1 The stabilizer. The flexible retractor spatula (white arrow) was attached to the Octopus universal retractor holder.

13 minutes 56 seconds (SD, 0 minute 53 seconds) in the stabilizer group and 15 minutes 25 seconds (SD, 4 minutes 11 seconds) in the nonstabilizer group $(p<0.05)$. The average venous microanastomosis times were 16 minutes $21 \mathrm{sec}-$ onds (SD, 2 minutes 4 seconds) in the stabilizer group and 22 minutes 16 seconds (SD, 2 minutes 8 seconds) in the nonstabilizer group $(p<0.05)$. The average outer diameters were $1.95(0.15) \mathrm{mm}$ in internal mammary arteries, 1.98 $(0.15) \mathrm{mm}$ in deep epigastric arteries, $1.76(0.36) \mathrm{mm}$ in internal mammary veins, and $1.78(0.35) \mathrm{mm}$ in deep epigastric veins. In the vessels, the right internal mammary artery diameter was $2.03(0.15) \mathrm{mm}$, the left internal mammary artery diameter was $1.84(0.07) \mathrm{mm}$, the right internal mammary vein diameter was $1.84(0.42) \mathrm{mm}$, and the left internal mammary vein diameter was $1.68(0.26) \mathrm{mm}$.

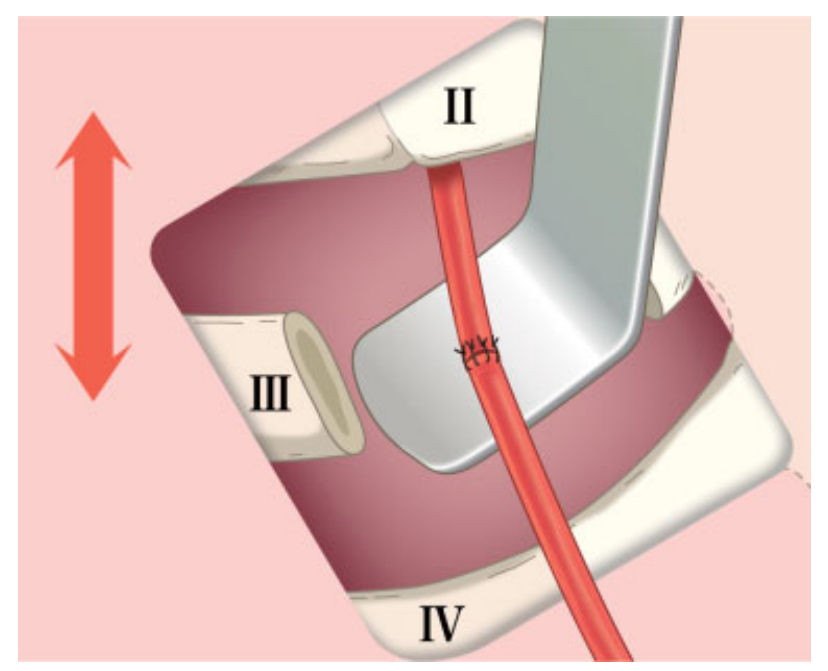

Fig. 2 The schema of the stabilizer. The tip of flexible retractor spatula was bent to 90 degrees for insertion at the space where the third costal rib was removed. Above the stabilizer, microanastomosis was performed without disturbance from respiratory fluctuation. 
28 Stabilizer for Microanastomoses of Internal Mammary Vessels Hamahata et al.

\section{Discussion}

With great advancements of microsurgical techniques, breast reconstruction using the free flap, especially the DIEP flap, is now globally popular. However, microanastomosis remains a stressful task for reconstructive surgeons. For microanastomoses of the DIEP flap, internal mammary vessels and thoracodorsal vessels can be used as recipient vessels, but the use of the internal mammary vessels has been reported to be clearly justified and preferred because of the highly pressurized system, consistent location, ease of accessibility, and flap positioning. ${ }^{4}$ We also prefer to use internal mammary vessels as recipient vessels, and these vessels were used as recipient vessels in all DIEP flap reconstruction cases in our institution. However, when using internal mammary vessels as recipient vessels, respiratory fluctuations may disturb surgeons, especially while viewing the vessels at high magnification during microanastomosis. Shifts in the focus field due to respiratory fluctuation commonly cause surgeons to suffer from asthenopia and even optic vertigo, and the quality of the precise microanastomosis may be decreased in worstcase scenarios. Also, it has been reported that microsurgeons have an extremely poor blink rate during microanastomosis, a rate which is lowered further during difficult sutures, thus causing surgeons to suffer from tired eyes and dry eyes due to concentration applied to microsurgery. ${ }^{5}$ Therefore, to reduce respiratory fluctuation during microsurgery, we developed the stabilizer for use during the procedure, which significantly reduced the microanastomosis time. And although subjective, surgeons' stress during microanastomosis was dramatically reduced during the operation by use of the stabilizer. In addition, water and blood that pool in the space where a rib is removed and which can distract microsurgeons from performing precise microanastomoses can be suctioned easily from under the stabilizer during microanastomoses.

A North American report indicated that the average diameters of internal mammary vessels were: right internal mammary artery, $2.52(0.51) \mathrm{mm}$; left internal mammary artery, 2.30 (0.55) mm; right internal mammary vein, 2.89 (0.56) $\mathrm{mm}$; and left internal mammary vein, 2.31 (0.48) $\mathrm{mm} .{ }^{6}$ However, in our study, the right internal mammary artery diameter was $2.03(0.15) \mathrm{mm}$, the left internal mammary artery diameter was $1.84(0.07) \mathrm{mm}$, the right internal mammary vein diameter was $1.84(0.42) \mathrm{mm}$, and the left internal mammary vein diameter was $1.68(0.26) \mathrm{mm}$. Our study patients were all Japanese and the average height was only $158.4 \mathrm{~cm}$, with vessel sizes being relatively smaller than the North American report. In our study, the left internal mammary vein was also smaller than the right internal mammary vein. This can be explained by human anatomy in that the left internal mammary vein frequently bifurcated at the second interspace and the right internal mammary vein bifurcated more inferiorly. 6

In our study, microanastomosis time was dramatically reduced in venous anastomosis compared with arterial anastomosis with the use of the stabilizer. The internal mammary vein has a small caliber, especially the left side, as described earlier, and a higher magnification power is required as compared with the artery. In addition, the venous wall is very thin and flimsy, which causes venous microanastomosis to be more difficult than arterial anastomosis. During microsurgery, respiratory fluctuation negatively affects the precision of microanastomosis, especially in cases of venous anastomosis. A vascular coupling device for microanastomosis is also one of the most useful devices for breast reconstruction. ${ }^{7,8}$ However, the procedure of putting the vessel wall on the device is not easy, and the fragile vessel wall will sometimes tear in small caliber vessels. We have rarely used the device in DIEP flap reconstruction.

\section{Conclusion}

We developed a stabilizer for microanastomosis of internal mammary vessels during DIEP flap breast reconstruction. The stabilizer significantly decreased the time of microanastomosis and reduced microsurgeons' stress.

\section{Acknowledgments}

The authors thank Jeffrey D. Meserve and Mayumi Noguchi for editorial assistance and figure drawing, respectively.

\section{References}

1 Holmström H. The free abdominoplasty flap and its use in breast reconstruction. An experimental study and clinical case report. Scand J Plast Reconstr Surg 1979;13(3):423-427

2 Robbins TH. Rectus abdominis myocutaneous flap for breast reconstruction. Aust N Z J Surg 1979;49(5):527-530

3 Koshima I, Soeda S. Inferior epigastric artery skin flaps without rectus abdominis muscle. Br J Plast Surg 1989;42(6):645-648

4 Nahabedian M. The internal mammary artery and vein as recipient vessels for microvascular breast reconstruction. Ann Plast Surg 2012;68(5):537-538

5 Payne CE. Tired eyes from microsurgery-it's blinking obvious!. J Reconstr Microsurg 2013;29(1):67-68

6 Feng LJ. Recipient vessels in free-flap breast reconstruction: a study of the internal mammary and thoracodorsal vessels. Plast Reconstr Surg 1997;99(2):405-416

7 Bodin F, Brunetti S, Dissaux C, et al. Venous coupler use for freeflap breast reconstructions: specific analyses of TMG and DIEP flaps. Microsurgery 2015;35(4):295-299

8 Sullivan SK, Dellacroce F, Allen R. Management of significant venous discrepancy with microvascular venous coupler.J Reconstr Microsurg 2003;19(6):377-380 